Case Report

\title{
De Novo Atypical Haemolytic Uremic Syndrome after Kidney Transplantation
}

\author{
Arnaud Devresse, ${ }^{1}$ Martine de Meyer, ${ }^{2}$ Selda Aydin, ${ }^{3}$ Karin Dahan, ${ }^{1,4,5}$ and Nada Kanaan (i) ${ }^{1}$ \\ ${ }^{1}$ Division of Nephrology, Cliniques Universitaires Saint-Luc, Université Catholique de Louvain, Brussels, Belgium \\ ${ }^{2}$ Division of Abdominal Surgery and Transplantation, Cliniques Universitaires Saint-Luc, Université Catholique de Louvain, \\ Brussels, Belgium \\ ${ }^{3}$ Division of Pathology, Cliniques Universitaires Saint-Luc, Université Catholique de Louvain, Brussels, Belgium \\ ${ }^{4}$ Division of Human Genetics, Cliniques Universitaires Saint-Luc, Université Catholique de Louvain, Brussels, Belgium \\ ${ }^{5}$ Center of Human Genetics, Institut de Pathologie et de Génétique, Gosselies, Belgium \\ Correspondence should be addressed to Nada Kanaan; nada.kanaan@uclouvain.be
}

Received 15 November 2017; Revised 23 January 2018; Accepted 14 February 2018; Published 14 March 2018

Academic Editor: Sophia Lionaki

Copyright @ 2018 Arnaud Devresse et al. This is an open access article distributed under the Creative Commons Attribution License, which permits unrestricted use, distribution, and reproduction in any medium, provided the original work is properly cited.

De novo thrombotic microangiopathy (TMA) can occur after kidney transplantation. An abnormality of the alternative pathway of complement must be suspected and searched for, even in presence of a secondary cause. We report the case of a 23-year-old female patient who was transplanted with a kidney from her mother for end-stage renal disease secondary to Hinman syndrome. Early after transplantation, she presented with 2 episodes of severe pyelonephritis, associated with acute kidney dysfunction and biological and histological features of TMA. Investigations of the alternative pathway of the complement system revealed atypical haemolytic uremic syndrome secondary to complement factor I mutation, associated with mutations in CD46 and complement factor $\mathrm{H}$ related protein genes. Plasma exchanges followed by eculizumab injections allowed improvement of kidney function without, however, normalization of creatinine.

\section{Background}

De novo thrombotic microangiopathy (TMA) has been reported to occur after kidney transplantation [1]. The pathogenic mechanisms are not well understood but are likely multifactorial with implication of specific features attributed to kidney transplantation [2]. However, the implication of a dysregulation in the alternative complement pathway may be underestimated [3].

\section{Case Report}

A 14-year-old female patient was admitted 9 years ago to our institution for acute pyelonephritis. Massive bilateral ureterohydronephrosis secondary to grade $\mathrm{V}$ ureterovesical reflux associated with a trabeculated bladder was evidenced (Figure 1). Neurologic investigations revealed no abnormality, leading to a diagnosis of Hinman syndrome, a very rare entity characterized by all features of a neurogenic bladder with external sphincter dyssynergia, but without evidence of any neurologic alteration [4]. After diagnosis, she had several uncomplicated urinary tract infections, necessitating selfcatheterization (importantly never associated with biological thrombocytopenia or haemolytic anemia), and reached endstage renal disease at age 23 when she underwent preemptive HLA semi-identical living-donor (her mother) kidney transplantation.

Her immunosuppressive regimen included Basiliximab induction, tacrolimus, mycophenolate mofetil, and steroids. She was discharged at day 11 with a normal plasma creatinine $(1.26 \mathrm{mg} / \mathrm{dl})$. At day 40 , she was admitted for intestinal occlusion due to adhesions requiring adhesiolysis. During hospitalization, she presented a severe pyelonephritis secondary to Pseudomonas aeruginosa (colony count $>100,000$ colonyforming units of bacteria per $\mathrm{mL}$ of urine with negative blood cultures but with increased level of C-reactive protein, 
TABLE 1: Laboratory findings.

\begin{tabular}{|c|c|c|c|}
\hline & Day $11^{\circ}$ & Day $40^{\circ}$ & Day $120^{\circ}$ \\
\hline C-reactive protein, $\mathrm{mg} / \mathrm{L}(N<5.0)$ & 33.0 & 117.0 & 452.0 \\
\hline Plasma creatinine, mg/dL ( $N$ : 0.60-1.30) & 1.21 & 2.73 & 5.5 \\
\hline Lactate dehydrogenase, IU/L $(N<250)$ & 376 & 722 & 517 \\
\hline Hemoglobin, g/dL (N: 12.2-15.0) & 9.6 & 7.7 & 7.0 \\
\hline Coombs test & NA & Negative & Negative \\
\hline Platelets count, per $\mu / \mathrm{L}(N: 150000-450000)$ & 417,000 & 51,000 & 96,000 \\
\hline Haptoglobin, g/L (N: 0.3-2.0) & NA & $<0.1$ & $<0.1$ \\
\hline Schistocytes count, \% of red blood cells & NA & 4 & 2 \\
\hline Tacrolimus trough level, ng/mL & 9.0 & 26.5 & 9.9 \\
\hline Anti-HLA antibody screening* & NA & Negative & Negative \\
\hline Complement C4, g/L (N: 0.1-0.4) & NA & 0.34 & 0.36 \\
\hline Complement C3, g/L (N: 0.9-1.8) & NA & 1.14 & 1.53 \\
\hline CMV (PCR), copies/mL & Undetected & Undetected & Undetected \\
\hline
\end{tabular}

${ }^{\circ}$ After kidney transplantation. ${ }^{*}$ Class I and class II anti-HLA antibody screening performed by single antigen bead assay. CMV: cytomegalovirus; HLA: human leukocyte antigen; IU: international unit; PCR: polymerase chain reaction; $N$ : normal value; NA: not available.

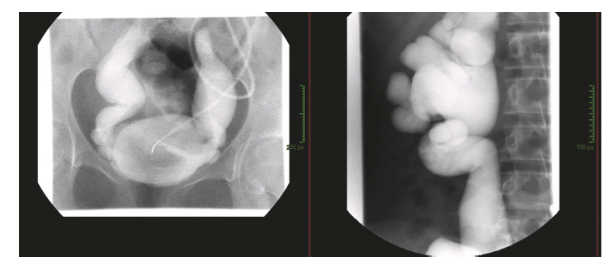

FIGURE 1: Retrograde cystography showing massive ureterovesical reflux and hydronephrosis.

features consistent with acute pyelonephritis), associated with acute renal failure (creatinine $2.73 \mathrm{mg} / \mathrm{dl}$ ), increased lactate dehydrogenase level, decreased haptoglobin, haemoglobin, and platelets level. Tacrolimus trough levels were elevated. Complement 3 and 4 levels were normal. Donor-specific antibodies (DSA) were negative (Table 1). She was treated with antibiotics, and a kidney biopsy performed 48 hours later was normal with no sign of acute rejection, acute pyelonephritis, or acute tubular necrosis. Interstitial fibrosis and tubular atrophy (IFTA) was scored 1. Because of the severity of the biological signs of thrombotic microangiopathy (TMA), she was treated with daily plasma exchanges with fresh frozen plasma for one week. Laboratory tests normalized except creatinine that remained elevated $(2.1 \mathrm{mg} / \mathrm{dl})$. At day 120 , the patient was admitted again for severe pyelonephritis secondary to Pseudomonas aeruginosa (>100,000 colonyforming units of bacteria per $\mathrm{mL}$ of urine with negative blood cultures) with acute renal failure (creatinine $5.5 \mathrm{mg} / \mathrm{dl}$ ) and the same biological picture (Table 1). A second kidney biopsy showed pathognomonic features of thrombotic microangiopathy (including a preglomerular arteriole of one glomerulus obstructed by a fresh thrombus and mesangiolysis, without argument for antibody-mediated rejection) (Figure 2). IFTA was scored 1. DSA were absent. Screening for secondary causes of TMA was negative (antiphospholipid syndrome, Shiga toxin, ADMATS13 deficiency or inhibitor, antinuclear factor, Coombs test, disseminated intravascular

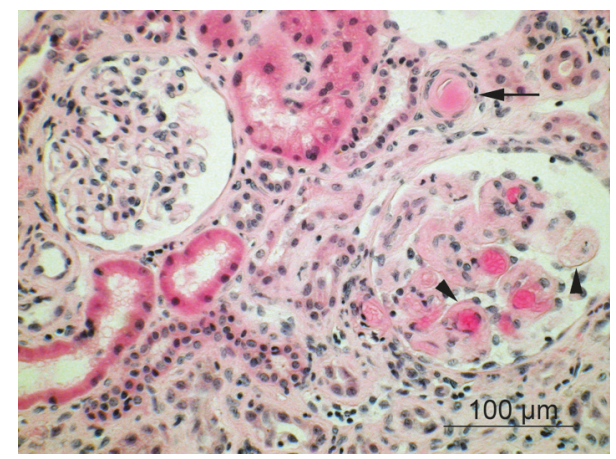

FIGURE 2: Histological examination showing thrombotic microangiopathy in a kidney biopsy from renal allograft at day 120 (hematoxylin and eosin). Microthrombi and lucent deposits (arrowheads) are observed in the glomerulus at the right side, with obstruction of a nearby arteriole by eosinophilic material (arrow). Notice the unaffected glomerulus on the left side of the microphotograph. There is no evidence of acute antibody-mediated rejection according to the 2015 Banff classification (g0, ptc0, and no C4d deposit by immunofluorescence (not shown)).

coagulation, HIV, CMV, pregnancy, hypertension, or occult infection). A genetic screening of the alternative complement pathway revealed a heterozygous mutation in complement factor I (CFI) gene c.148C $>\mathrm{G}$ (p.(Pro50AIa)), associated with a heterozygous variant of membrane cofactor protein $C D 46$ (c-366A) and a homozygote deletion of complement factor $\mathrm{H}(\mathrm{CFH})$ related protein $C F H R 1$ and CFHR3 genes. Anti-FH antibody screening was negative. The patient received two plasma exchanges with fresh frozen plasma while waiting to have fast access to anti-C5 antibody. Eculizumab was then started using the recommended doses $(900 \mathrm{mg} /$ week for 1 month and then $1200 \mathrm{mg} / 2$ weeks). She was treated for 10 months (the period allowed by our legislation).

Creatinine level stabilized around $2 \mathrm{mg} / \mathrm{dl}$. An allograft biopsy performed 3 months after initiation of eculizumab 
showed no sign of TMA but a progression of IFTA score to 2. She did not experience any infectious event or biological signs of TMA under eculizumab and is currently doing fine 3 months after treatment cessation.

Screening of the mother revealed the same mutations in CFI and CFHR1-CFHR3. Her pre-kidney-donation workup was normal and the postnephrectomy evolution was uneventful. Genetic screening was proposed to other members of family (patient's father and brother) but has not been performed yet.

\section{Discussion}

TMA is a pathologic description, clinically characterized by an association of thrombocytopenia, microangiopathic haemolytic anemia and organ injury [1]. After solid organ transplantation (including kidney, liver, pancreas, lung, and heart) or bone marrow transplantation, de novo TMA had been reported to occur $[1,5,6]$. In the kidney transplantation setting, de novo TMA classically occurred in the 6 first months after kidney transplantation [7] with an incidence between $0.8 \%$ to $14 \%[3,7]$. If the pathogenic mechanisms of de novo TMA are not well understood, they are likely to be multifactorial with ischemia-reperfusion injury, antibody-mediated rejection, viral infection such as cytomegalovirus and immunosuppressant drugs, especially calcineurin inhibitors (CNI), contributing to an "endothelial damaging milieu" [2]. In many cases, supportive treatment and addressing the precipitating factors (CNI dose reduction, CNI withdrawal, treatment of acute antibodymediated rejection, and viral infections) are sufficient to stop TMA [1]. However, for some patients, this strategy does not lead to an improvement of TMA. For those patients, a complement-mediated TMA secondary to a dysregulation of the alternative complement pathway, classically called atypical haemolytic and uremic syndrome (a-HUS), should be suspected. a-HUS is a rare disorder due to genetic mutation of the alternative complement pathway [8]. These mutations can be found in the regulatory genes (CFH, CD46, CFI, Thrombomodulin) or in the activatory genes (factor $B, C 3$ ). a-HUS can also be secondary to anti-CFH antibodies [8]. A trigger event such as infection or pregnancy is believed to precipitate aHUS in a susceptible individual. Making the genetic diagnosis of a-HUS before kidney transplantation is crucial: first, the risk of recurrence after kidney transplantation depends on whether the mutant complement factor is membrane-bound (low risk) or circulating (high risk) [9]; second, the introduction of eculizumab, a terminal complement inhibitor, as preventive treatment, has dramatically improved the risk of a-HUS recurrence after kidney transplantation leading to a huge improvement in the allograft survival in these patients [10-12].

In the setting of de novo TMA after kidney transplantation, the implication of a dysregulation in the alternative complement pathway may be underestimated as suggested by one series of de novo TMA after kidney transplantation published by le Quintrec et al. [3]. In a cohort of 24 deceaseddonor kidney transplant recipients who experienced de novo TMA after kidney transplantation and who had systematic screening for mutations in genes encoding $\mathrm{CFH}, \mathrm{CFI}$, and CD46, 7/24 patients were found to have a mutation: $1 \mathrm{CFH}, 4$ $\mathrm{CFI}$, and $2 \mathrm{CFH}$ and CFI. Mutations in CFI are heterozygous in most patients. Interestingly, $30 \%$ of patients with CFI mutation were found to have an additional mutation in genes known to be susceptible risk factors for a-HUS. The diagnosis of a-HUS in our patient before transplantation was not suspected. Indeed, she did not show any signs of TMA despite several episodes of urinary tract infections. Her mother also never experienced signs of TMA neither before transplantation (despite 2 pregnancies) nor after kidney donation. The genetics of these mutations is highly complex with a penetrance around 50\% [13]. Familial studies suggest a monoallelic autosomal or pseudoautosomal mode of inheritance $[14,15]$. Moreover, within families, affected persons may also show different symptoms and ages at onset of the disease [13]. This highly suggests that most a-HUSassociated genetic variants predispose to rather than cause the disease and that triggers are necessary to develop symptoms as for our patient who exacerbated a-HUS symptoms only in the presence of several pathologic conditions: kidney transplantation, immunosuppression, and infection.

Our patient improved her allograft renal function after eculizumab initiation suggesting that, besides preventing recurrence after transplantation, it can be efficient to reverse the fate of renal function in de novo a-HUS occurring after kidney transplantation as demonstrated in a-HUS occurring in the nontransplant setting [11]. Treatment duration of eculizumab is controversial. Despite early recommendations for a lifelong therapy, there is no evidence supporting this attitude [16]. Recently, reports have suggested that, in native kidneys, eculizumab therapy may be discontinued after remission has been achieved, with a prompt resumption of therapy in cases of relapse. Wijnsma et al. reported 20 nonkidney-transplant patients, in whom a restrictive treatment in time followed by a TMA monitoring appeared safe and effective [17]. In kidney transplant recipients, Duineveld et al. reported recently a case series including 17 patients with a-HUS who underwent living kidney transplantation without prophylactic eculizumab. A monitoring strategy was applied and was successful, as only one patient experienced recurrence, which was successfully treated [18]. The good outcomes in this report may be due to the fact that (1) all living donors were genotyped, (2) cold ischemia time was short, and (3) low targets of tacrolimus were used [19]. In our patient, eculizumab was discontinued after 10 months due to limitations imposed by our national reimbursement policy. Currently, 3 months after therapy cessation, she is doing fine with a monthly biological screening. Prospective studies including larger cohorts of kidney transplant recipients with a long follow-up are required to assess whether eculizumab prophylaxis should be restricted to specific profiles and to assess treatment duration.

In conclusion, our case highlights the importance of the following: (1) a genetic screening in de novo TMA after kidney transplantation, (2) identifying the underlying mutation allowing treatment that can potentially reverse the fate of renal function, (3) familial screening and counselling in the 
context of living donation in case of suspected biological TMA in the donor and/or the recipient.

\section{Conflicts of Interest}

The authors declare that they have no conflicts of interest.

\section{References}

[1] V. Brocklebank, K. M. Wood, and D. Kavanagh, "Thrombotic microangiopathy and the kidney," Clinical Journal of the American Society of Nephrology, 2017.

[2] J. Zuber, M. Le Quintrec, H. Morris, V. Frémeaux-Bacchi, C. Loirat, and C. Legendre, "Targeted strategies in the prevention and management of atypical HUS recurrence after kidney transplantation," Transplantation Reviews, vol. 27, no. 4, pp. 117125, 2013.

[3] M. le Quintrec, A. Lionet, N. Kamar et al., "Complement mutation-associated de novo thrombotic microangiopathy following kidney transplantation," American Journal of Transplantation, vol. 8, no. 8, pp. 1694-1701, 2008.

[4] S. B. Bauer, “The Hinman Syndrome," The Journal of Urology, vol. 197, no. 2, pp. S132-S133, 2017.

[5] A. Verbiest, J. Pirenne, and D. Dierickx, "De novo thrombotic microangiopathy after non-renal solid organ transplantation," Blood Reviews, vol. 28, no. 6, pp. 269-279, 2014.

[6] C. Ponticelli and G. Banfi, "Thrombotic microangiopathy after kidney transplantation," Transplant International, vol. 19, no. 10, pp. 789-794, 2006.

[7] A. A. Satoskar, R. Pelletier, P. Adams et al., "De novo thrombotic microangiopathy in renal allograft biopsies-role of antibodymediated rejection," American Journal of Transplantation, vol. 10, no. 8, pp. 1804-1811, 2010.

[8] M. Noris and G. Remuzzi, "Atypical hemolytic-uremic syndrome," The New England Journal of Medicine, vol. 361, no. 17, pp. 1676-1687, 2009.

[9] M. Okumi and K. Tanabe, "Prevention and treatment of atypical haemolytic uremic syndrome after kidney transplantation," Nephrology, vol. 21, pp. 9-13, 2016.

[10] F. Fakhouri, J. Zuber, V. Frémeaux-Bacchi, and C. Loirat, "Haemolytic uraemic syndrome," The Lancet, vol. 390, no. 10095, pp. 681-696, 2017.

[11] C. M. Legendre, C. Licht, P. Muus et al., "Terminal complement inhibitor eculizumab in atypical hemolytic-uremic syndrome," The New England Journal of Medicine, vol. 368, no. 23, pp. 21692181, 2013.

[12] A. Kumar, Z. Stewart, A. Reed et al., "Successful prophylactic use of eculizumab in ahus kidney transplant patients: a report of 9 cases," American Journal of Transplantation, vol. 16, supplement 3, 2016.

[13] F. Bu, N. Borsa, A. Gianluigi, and R. J. H. Smith, "Familial atypical hemolytic uremic syndrome: a review of its genetic and clinical aspects," Clinical and Developmental Immunology, vol. 2012, Article ID 370426, 9 pages, 2012.

[14] J. Caprioli, M. Noris, S. Brioschi et al., "Genetics of HUS: the impact of MCP, CFH, and IF mutations on clinical presentation, response to treatment, and outcome," Blood, vol. 108, no. 4, pp. 1267-1279, 2006.

[15] D. Kavanagh, A. Richards, and J. Atkinson, "Complement regulatory genes and hemolytic uremic syndromes," Annual Review of Medicine, vol. 59, pp. 293-309, 2008.
[16] T. H. Goodship, H. T. Cook, F. Fakhouri et al., "Atypical hemolytic uremic syndrome and C3 glomerulopathy: conclusions from a "Kidney Disease: improving Global Outcomes" (KDIGO) Controversies Conference," Kidney International, vol. 99, no. 3, pp. 539-551, 2017.

[17] K. L. Wijnsma, C. Duineveld, E. B. Volokhina, L. P. van den Heuvel, N. C. van de Kar, and J. F. Wetzels, "Safety and effectiveness of restrictive eculizumab treatment in atypical haemolytic uremic syndrome," Nephrology Dialysis Transplantation, 2017.

[18] C. Duineveld, J. C. Verhave, S. P. Berger, N. C. A. J. van de Kar, and J. F. M. Wetzels, "Living donor kidney transplantation in atypical hemolytic uremic syndrome: a case series," American Journal of Kidney Diseases, vol. 70, no. 6, pp. 770-777, 2017.

[19] M. Noris, P. Ruggenenti, and G. Remuzzi, "Kidney transplantation in patients with atypical hemolytic uremic syndrome: a therapeutic dilemma (or not)?" American Journal of Kidney Diseases, vol. 70, no. 6, pp. 754-757, 2017. 


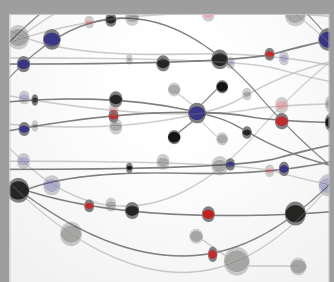

The Scientific World Journal
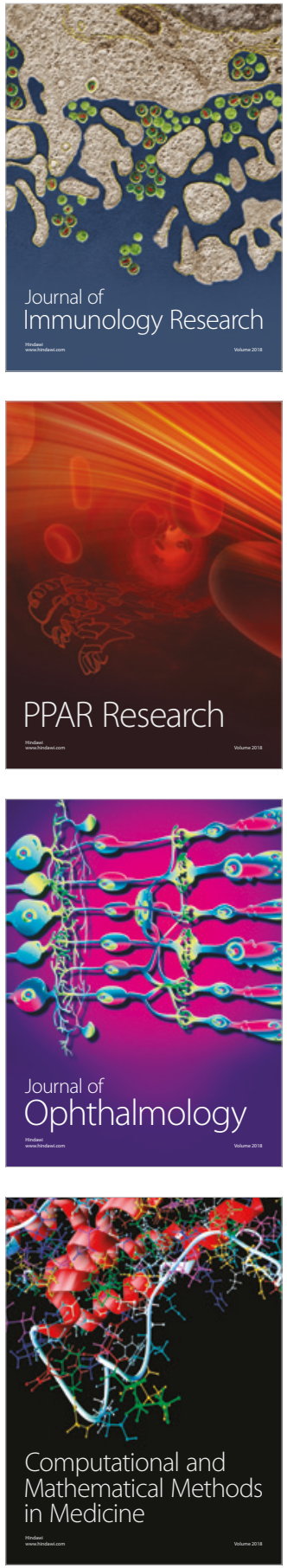

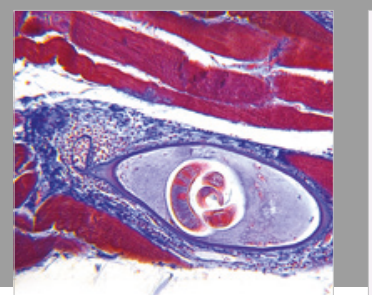

Gastroenterology Research and Practice

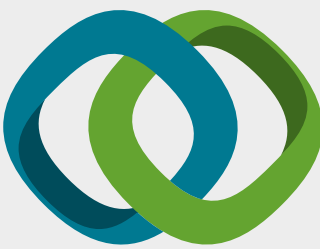

\section{Hindawi}

Submit your manuscripts at

www.hindawi.com
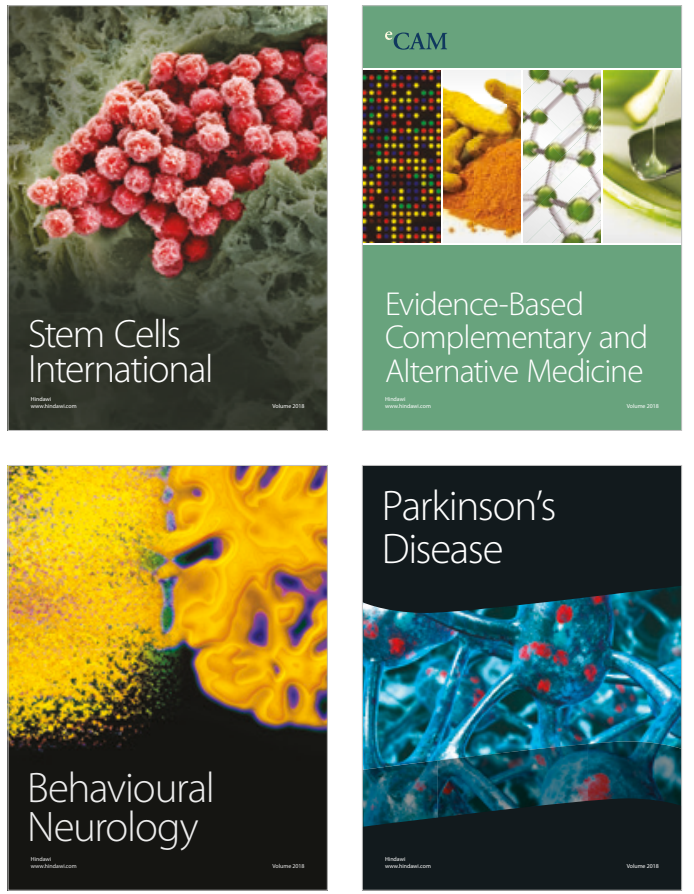

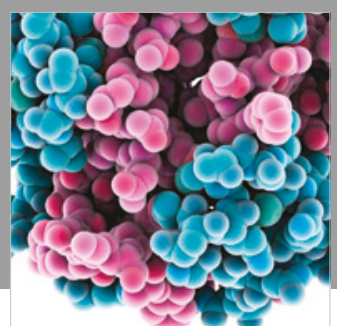

ournal of

Diabetes Research

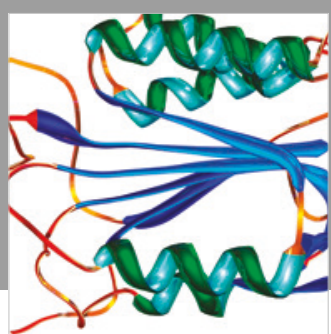

Disease Markers
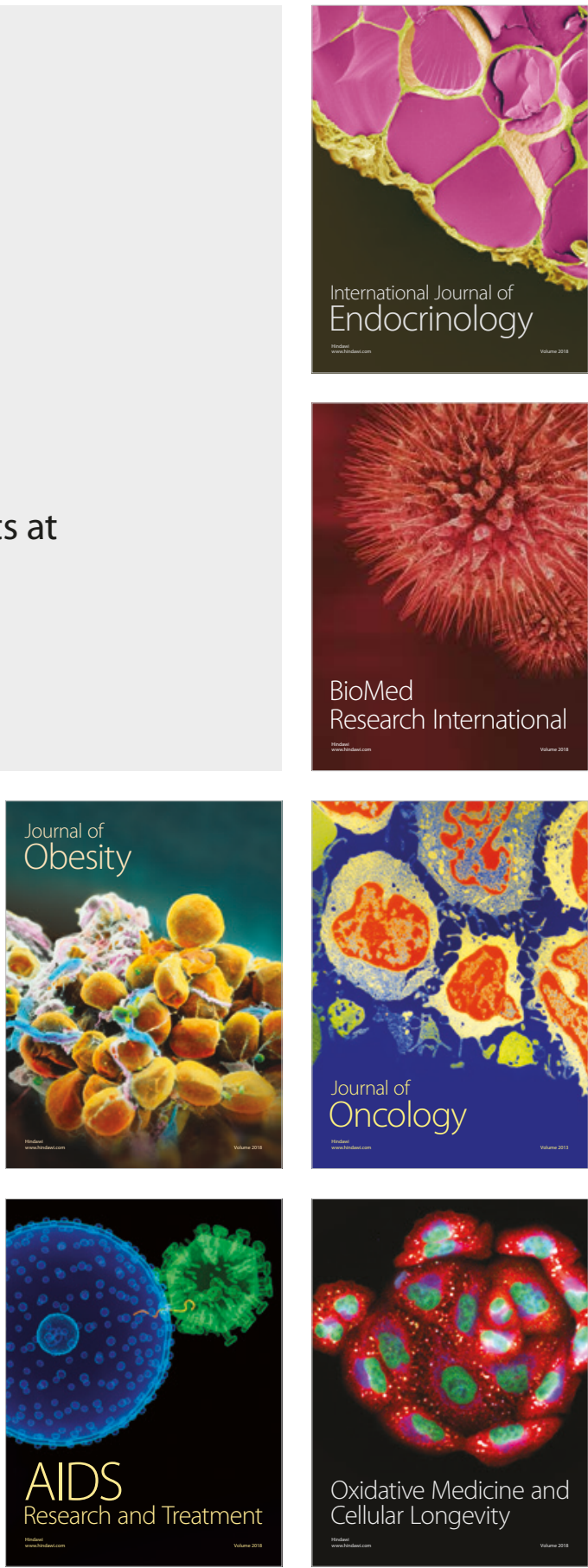